03

\title{
Метод расчета нестационарного теплового потока по сигналу датчика на основе анизотропных термоэлементов из монокристалла висмута
}

\author{
(С) П.А. Попов, С.В. Бобашев, Б.И. Резников, В.А. Сахаров \\ Физико-технический институт им. А.Ф. Иоффре РАН, Санкт-Петербург, \\ Россия \\ E-mail: pavel.popov@mail.ioffe.ru
}

Поступило в Редакцию 12 сентября 2017 г.

Предложен метод расчета нестационарного теплового потока по электрическому сигналу датчика на основе анизотропных термоэлементов из монокристалла висмута. Он позволяет приближенно вычислить значение теплового потока в диапазоне от $\sim 1 \mu \mathrm{s}$ до времени выхода на стационарный режим. Тестирование метода показало, что при регистрации тепловых потоков малой длительности $(t \sim 1-10 \mathrm{~ms})$ датчиками на основе термоэлементов с соотношением сторон $>25$ погрешность не превышает нескольких процентов.

DOI: 10.21883/PJTF.2018.08.45960.17036

В экспериментальной газодинамике наряду с традиционными средствами измерения теплового потока, такими как тонкопленочные датчики сопротивления и коаксиальные термопары [1], в последнее время получил распространение новый тип датчиков на основе поперечного эффекта Зеебека. В частности, датчиками такого типа являются ALTP (atomic layer thermopile) с чувствительным элементом из анизотропной пленки $\mathrm{YBCO} / \mathrm{CuO}_{2}[2]$ и ГДТП (градиентный датчик теплового потока) 
с анизотропными термоэлементами из монокристалла висмута [3]. Они обладают высокой чувствительностью, малым временем отклика на тепловое воздействие и активно применяются в различных теплофизических экспериментах, в том числе в импульсных высокоэнтальпийных установках [4-6].

Метод обработки результатов измерений датчиком на основе эффекта Зеебека зависит от теплового режима, в котором он находился в процессе регистрации. Время установления стационарного теплового режима $t$ в сборке чувствительный элемент-подложка может быть определено с помощью соотношения [7]

$$
\mathrm{Fo}=\frac{\eta(1+\chi \eta)+\operatorname{Bi}\left[\eta+1 / 2 \chi\left(1+\eta^{2}\right)\right]}{\operatorname{Bi} \chi},
$$

где $\mathrm{Fo}=\frac{a t}{h}, \mathrm{Bi}=\frac{\alpha s}{\lambda_{2}}, \eta=\frac{s}{h} \sqrt{\frac{a_{1}}{a_{2}}}, \chi=\sqrt{\frac{\lambda_{1} C_{1} \rho_{1}}{\lambda_{2} C_{2} \rho_{2}}}, a=\frac{\lambda}{C \rho} ; \alpha-$ коэффициент теплоотдачи от тыльной стороны подложки; $h, s-$ толщины термоэлемента и подложки; $\lambda-$ теплопроводность; $C-$ теплоемкость; $\rho$ - плотность. Оно определяет границу применимости стационарной калибровки для расчета теплового потока: $q=k U$, где $k-$ калибровочный коэффициент, а $U$ - электрический сигнал датчика. B ALTP с толщиной чувствительного элемента $\sim 1 \mu \mathrm{m}$ время установления стационарного режима составляет $\sim 1 \mu \mathrm{s}$ [2], что позволяет использовать его для прямого измерения теплового потока в газодинамическом эксперименте. Минимальная толщина термоэлементов в ГДТП составляет $200 \mu \mathrm{m}$, при этом время выхода на стационарный режим достигает $\sim 1 \mathrm{~s}[8,9]$. При меньших характерных временах изменения теплового потока применение стационарной калибровки приводит к качественно неверным результатам [10] и необходим иной подход к обработке результатов измерений.

В настоящей работе предлагается метод расчета нестационарного теплового потока по сигналу датчика на основе анизотропных термоэлементов из монокристалла висмута (ГДТП). Он позволяет приближенно рассчитать тепловой поток в диапазоне от $\sim 1 \mu \mathrm{s}$ до времени выхода на стационарный тепловой режим.

Рассмотрим модель датчика, состоящую из термоэлемента прямоугольной формы $(1)$ длиной $l$ и толщиной $h$, расположенного на изоляционной подложке из слюды (2) толщиной $s$ (рис. 1). Через рабочую поверхность термоэлемента $y=h$ проходит тепловой поток $q_{h}$,

Письма в ЖТФ, 2018, том 44, вып. 8 


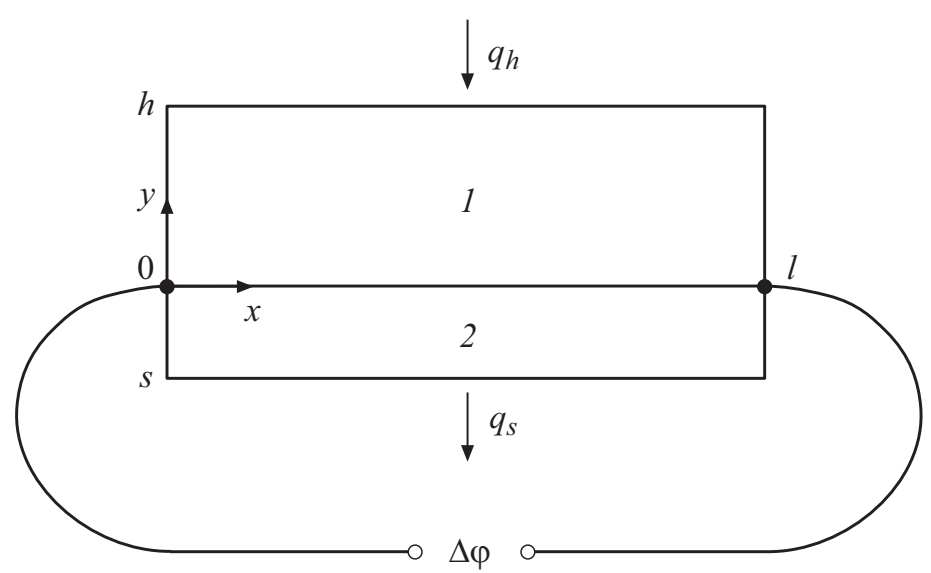

Рис. 1. Модель теплового датчика на основе анизотропного термоэлемента. 1 - анизотропный термоэлемент, 2 - подложка.

боковые поверхности термоэлемента и подложки $x=0$ и $x=l$ теплоизолированы. На тыльной поверхности подложки $y=s$ осуществляется конвективный теплообмен $q_{s}=\alpha_{s}\left(T_{s}-T_{e}\right)$, где $\alpha_{s}-$ коэффициент теплоотдачи, $T_{s}$ и $T_{e}$ - температуры подложки и окружающей среды. На всех поверхностях термоэлемента задается условие электроизоляции $j_{n}=0$. Создаваемое напряжение $\Delta \varphi$ снимается в точках, расположенных на тыльной поверхности термоэлемента $y=0$.

Далее используются два варианта математической модели датчика. В первой модели (полной) распределение температуры $T(t, x, y)$ и потенциала $\varphi(t, x, y)$ находится из численного решения системы уравнений [11]

$$
\begin{gathered}
C \rho \frac{\partial T}{\partial t}+\operatorname{divw}=0, \\
\operatorname{divj}=0,
\end{gathered}
$$

где $\mathbf{w}=\mathbf{q}+\varphi \mathbf{j}-$ плотность потока энергии, $\mathbf{q}=-\lambda \nabla T+\alpha T \mathbf{j}$, $\mathbf{j}=-\sigma \nabla \varphi-\sigma \alpha \nabla T-$ плотности потока тепла и электрического тока, $T-$ температура, $\varphi-$ электрический потенциал, $\lambda, \sigma, \alpha-$ тензоры теплопроводности, электропроводности и термоэдс. Данная модель ис-

Письма в ЖТФ, 2018, том 44, вып. 8 
пользуется для решения прямой задачи - точного расчета напряжения $\Delta \varphi$ при нагреве датчика тепловым потоком $q_{h}$.

Вторая модель (упрощенная) используется для решения обратной задачи - приближенного расчета перепада температуры $\left(T_{h}-T_{0}\right)$ между рабочей $y=h$ и тыльной $y=0$ поверхностями термоэлемента по известному напряжению $\Delta \varphi$. В модели предполагается, что термоэлементы достаточно длинные, а распределение температуры одномерно: $T=T(t, y)$. Учитываются только компонента $\alpha_{x y}$ тензора термоэдс и компонента $\lambda_{y y}$ тензора теплопроводности висмута. Теплоемкость и плотность термоэлемента и подложки в обеих моделях одинаковы. При данных предположениях справедлива формула Томсона $\Delta \varphi=\alpha_{x y} \frac{l}{h}\left(T_{h}-T_{0}\right)[11]$. При $l / h>10$ она обладает приемлемой точностью [9]. В настоящей работе формула Томсона используется для определения температуры рабочей поверхности термоэлемента $T_{h}$ по известному из эксперимента электрическому сигналу датчика $\Delta \varphi$. Далее, искомый тепловой поток $q_{h}$ может быть определен из решения уравнения теплопроводности $C \rho \frac{\partial T}{\partial t}=\frac{d}{d y}\left(\lambda_{y y} \frac{d T}{d y}\right)$ с граничным условием $T_{h}=\frac{\Delta \varphi h}{\alpha_{x y} l}+T_{0}$ для двухслойной структуры термоэлемент-подложка. Особенностью данного выражения является наличие неизвестной температуры тыльной поверхности термоэлемента $T_{0}$. Для расчета $T_{h}$ можно воспользоваться итерационной процедурой решения уравнения теплопроводности с граничным условием $T_{h}^{i+1}=\frac{\Delta \varphi h}{\alpha_{x y} l}+T_{0}^{i}$, где $i-$ номер итерации. В качестве нулевого приближения $T_{h}^{0}$ принимается известная величина $\left(T_{h}-T_{0}\right)=\frac{\Delta \varphi h}{\alpha_{x y} l}$, а $T_{0}^{0}$ задается равной нулю. Условием окончания итераций можно считать достижение необходимой точности расчета температуры рабочей поверхности $\left(T_{h}^{i+1}-T_{h}^{i}\right)<\varepsilon$.

Тестирование предлагаемого метода проводилось на модельной задаче нагрева рабочей поверхности датчика импульсным тепловым потоком $q_{h}$. По заданному тепловому потоку с помощью первой модели рассчитывалось напряжение $\Delta \varphi$, рассматриваемое в качестве сигнала датчика, по которому восстанавливался тепловой поток. Поскольку метод является приближенным, необходимо оценить погрешности расчета теплового потока для термоэлементов, используемых в реальных датчиках (ГДТП). Рассмотрим два варианта типичных термоэлементов из монокристалла висмута длиной $l=2$ и $5 \mathrm{~mm}$, толщиной $h=0.2 \mathrm{~mm}$ $(l / h=10,25)$, расположенных на подложке из слюды такой же тол-

Письма в ЖТФ, 2018, том 44, вып. 8 
щины. Представляет интерес нагрев датчика тепловым потоком $q_{h}$ с различными характерными временами. В качестве масштаба выберем время, в течение которого температура тыльной поверхности термоэлемента остается неизменной, а распределение температуры остается близким к одномерному. Для термоэлементов толщиной $h=200 \mu \mathrm{m}$ оно составляет $\sim 0.5 \mathrm{~ms}$ [12]. Рассмотрим импульсный нагрев с характерным временем $t_{0}=50 \mu \mathrm{s}$ и $5 \mathrm{~ms}$.

На рис. 2 показаны заданный тепловой поток $q_{h}$ и рассчитанный с помощью предлагаемого метода для термоэлементов с соотношением сторон $l / h=10$ и 25. При малом характерном времени импульса (фрагмент $a$ ) распределение температуры практически одномерно, и даже в случае короткого термоэлемента погрешность расчета теплового потока не превышает 10\%. Наибольшее расхождение наблюдается в период максимальной интенсивности нагрева, далее кривые практически совпадают. При увеличении характерного времени импульса и продолжительности нагрева термоэлемента кривые ведут себя иначе. На начальном этапе они совпадают. Начиная с некоторого момента времени, когда отклонение от одномерного распределения температуры становится существенным, различие между кривыми увеличивается, достигая максимального значения при выходе на стационарный режим. Так же как и в предыдущем случае, увеличение длины термоэлементов повышает точность расчета теплового потока.

На рис. 3 показана зависимость максимальной погрешности расчета теплового потока с помощью предлагаемого метода от соотношения сторон термоэлемента $l / h$. Она соответствует стационарному тепловому режиму, когда распределение температуры в наибольшей степени отличается от одномерного.

Как показали проведенные тестовые расчеты, при небольших временах $(t \sim 10 \mathrm{~ms})$ для достижения сходимости процедуры достаточно нескольких итераций. При существенном увеличении времени количество итераций возрастает, при этом накапливаются ошибки численного расчета, что может привести к расхождению процедуры или повышению погрешности величины теплового потока.

В работе предложен метод расчета нестационарного теплового потока по электрическому сигналу датчика на основе анизотропных термоэлементов из монокристалла висмута. Проведенное тестирование показало, что при регистрации тепловых потоков малой длительности $(t \sim 1-10 \mathrm{~ms})$ датчиками с достаточно длинными термоэлементами

Письма в ЖТФ, 2018, том 44, вып. 8 

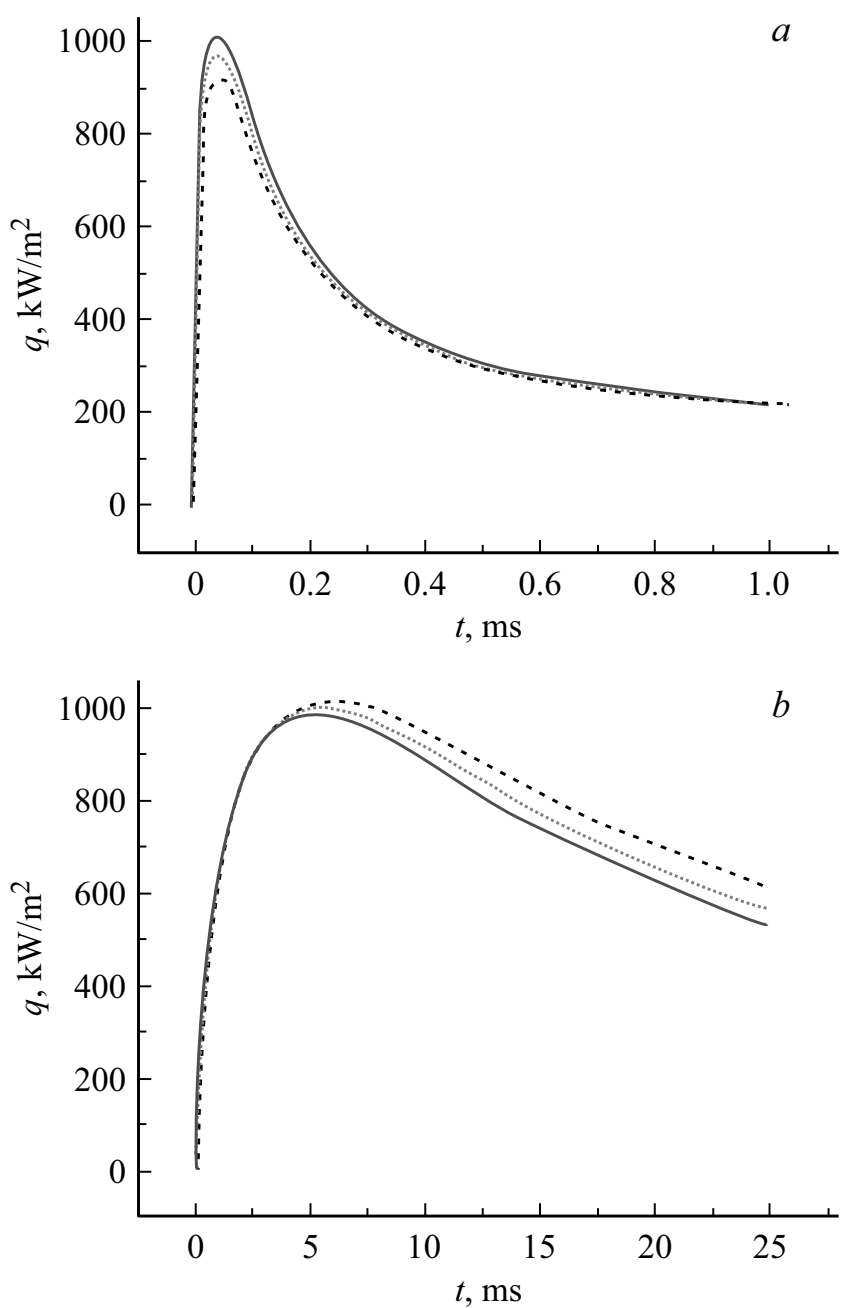

Рис. 2. Заданный тепловой поток $q_{h}$ (сплошная линия) и рассчитанный с помощью предлагаемой методики для термоэлементов длиной $l=2 \mathrm{~mm}$ (штриховая линия) и $5 \mathrm{~mm}$ (пунктирная линия). Характерное время импульса $t_{0}=50 \mu \mathrm{s}(a)$ и $5 \mathrm{~ms}(b)$. 


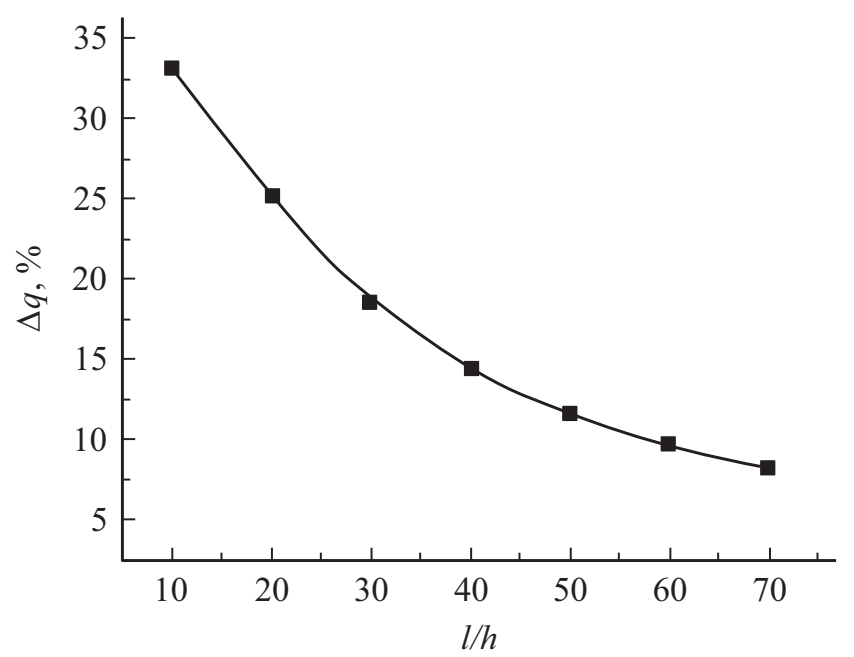

Pис. 3. Зависимость максимальной погрешности расчета теплового потока от соотношения сторон термоэлемента $l / h$.

$(l / h>25)$ погрешность не превышает нескольких процентов. Для повышения точности измерения тепловых потоков с большими характерными временами необходимо применять датчики с максимально возможным соотношением сторон термоэлемента.

\section{Список литературы}

[1] Hollis B.R., Prabhu D.K., MacLean M., Dufrene A. // J. Thermophys. Heat Transfer. 2017. V. 31. N 3. P. 712-731.

[2] Roediger T., Knauss H., Bountin D., Smorodsky B., Maslov A., Srulijes J. // J. Spacecraft Rockets. 2009. V. 46. N 2. P. 255-265.

[3] Mityakov A.V., Sapozhnikov S.Z., Mityakov V.Y, Snarskii A.A., Zhenirovsky M.I., Pyrhonen J.J. // Sensors Actuators A: Physical. 2012. V. 176. P. 1-9.

[4] Bobashev S., Golovachov Y., Chernyshev A., Kurbatov G., Mende N., Sakharov V., Schmidt A., Van Wie D. // 46th AIAA Aerospace Sciences Meeting. Reno, Nevada, 2008. P. 2008-1094.

Письма в ЖТФ, 2018, том 44, вып. 8 
[5] Bobashev S., Mende N., Ponjaev S., Popov P., Sakharov V., Sapozhnikov S., Mityakov V., Mityakov A., Van Wie D. // 47th AIAA Aerospace Sciences Meeting. Orlando, Florida. 2009. P. 2009-1041.

[6] Marineau E., Lewis D., Smith M., Lafferty J., White M., Amar A. // 51st AIAA Aerospace Sciences Meeting. Grapevine, Texas, 2013. V. 6. P. 4691-4716.

[7] Геращенко O.A. Основы теплометрии. Киев: Наук. думка, 1971. 188 с.

[8] Сапожников С.3., Митяков В.Ю., Митяков А.В. Основы градиентной теплометрии. СПб.: Изд-во Политехн. ун-та, 2012. 203 с.

[9] Попов П.А., Бобашев С.В., Резников Б.И., Сахаров В.А. // Письма в ЖТФ. 2017. T. 43. B. 7. C. $24-31$.

[10] Бобамев С.В., Менде Н.П., Попов П.А., Резников Б.И., Сахаров В.А., Сапожников С.З., Митяков В.Ю., Митяков А.В., Бунтин Д.А., Маслов А.А., Кнаусс Х., Редигер Т. // Письма в ЖТФ. 2009. Т. 35. В. 5. С. 36-42.

[11] Rowe D.M. CRC Handbook of thermoelectrics: macro to nano. CRC Press / Taylor \& Francis, 2006. $1014 \mathrm{p}$.

[12] Резников Б.И., Сахаров В.А., Штейнберг А.С. // Письма в ЖТФ. 2008. T. 34. B. 5. C. $28-33$. 